\title{
Editorial
}

\section{Smart Processing for Systems under Uncertainty or Perturbation}

\author{
Sanghyuk Lee ${ }^{1, *(1)}$, Mihail Popescu ${ }^{2}\left(\mathbb{D}\right.$ and Eneko Osaba ${ }^{3}(\mathbb{D}$ \\ 1 Department of Mechatronics and Robotics, School of Advanced Technology, Xi'an Jiaotong-Liverpool \\ University, Suzhou 215123, China \\ 2 Department of Health Management and Informatics, School of Medicine, University of Missouri, \\ Columbia, MO 65211, USA; popescum@health.missouri.edu \\ 3 TECNALIA, Basque Research and Technology Alliance (BRTA), 48160 Derio, Spain; \\ eneko.osaba@tecnalia.com \\ * Correspondence: sanghyuk.lee@xjtlu.edu.cn; Tel.: +86-512-8816-1415
}

Citation: Lee, S.; Popescu, M.; Osaba, E. Smart Processing for Systems under Uncertainty or Perturbation. Electronics 2022, 11, 680. https://doi.org/10.3390/ electronics 11050680

Received: 7 February 2022 Accepted: 8 February 2022 Published: 23 February 2022

Publisher's Note: MDPI stays neutral with regard to jurisdictional claims in published maps and institutional affiliations.

Copyright: (C) 2022 by the authors. Licensee MDPI, Basel, Switzerland. This article is an open access article distributed under the terms and conditions of the Creative Commons Attribution (CC BY) license (https:// creativecommons.org/licenses/by/ $4.0 /)$.

\section{Introduction}

Recently, systems have become more flexible and smarter in their implementation and functions. Such a technology trend challenges us to study smart processing for both systems and society. As systems have become more complex, larger in scale, and more high-dimensional, huge amounts of data processing have become necessary. For this reason, both internal and external systems sustainability have been emphasized. Researchers have thus been required to tackle these problems by considering uncertainty and perturbation. Together with smart device development, sound algorithms are also needed, such as deep learning techniques or the convergence idea. As computer specifications have increased and hardware such as smart devices and the internet of things (IoT) have become more accessible, we are now better able to meet these challenges. The necessity of this research can be summarized as follows:

- $\quad$ Recently, systems are being integrated into more complex scenarios and their functionalities are being augmented. This environment has led to a need for smarter management and control architectures, with the aim of maintaining the whole process properly under uncertainty or perturbation.

- The specifications, capacity and functionalities of existing systems need to be extended in order to be scalable. In this case, a smarter and more flexible methodology is required to maintain system performance.

- The usability of these systems needs to be extended into engineering environments and other areas. This should not be limited to industrial and engineering systems; these research experiences and methodologies can be applied to other areas.

This editorial introduces a Special Issue organized around the latest advances and applications in smart processing for systems under uncertainty or perturbation from two perspectives: new theoretical advances and their application to complex problems. This Special Issue provides the opportunity for researchers to share their novel research ideas from the relevant areas. In this open forum, we have received papers from various research fields: computer science, electrical engineering, mechanical engineering, education, and even the social sciences. After a strict review process of all the submissions received, ten different high-quality papers have been accepted. The accepted papers cover a large range of aspects, which are presented and discussed in the remainder of this manuscript. We conclude this brief editorial with a closing note, in which we call for further efforts around this exciting topic.

\section{An Overview of the Special Issue}

As mentioned, the contributions included in this issue address a wide variety of related topics, such as algorithm implementation, data processing and analysis and management under uncertain circumstances. A brief description of the published papers is given below. 
Research on algorithm implementation was carried out in [1-3]. Yang et al. propose two deep neural networks based on the attention mechanism to improve a sequence-topoint learning model. Their results show that the proposed mechanisms effectively improve performance. Next, Zeng et al. provide an interactive evolutionary design for product styling from the perspective of the cognitive association of the users. In the paper, they code the profiles of typical cases based on organized perceptual evaluation databases. The simplification of the process of creating associations between similar patterns is proposed based on explicit and implicit distances. Their experimental results show that this reduces the initial and total evaluation time. Finally, Jo et al. propose a recommendation deep learning algorithm for the fashion industry. They emphasize intelligent recommendation based on deep learning, which consists of a sketch-product fashion retrieval model and a vector-based model. The performance shown in the paper provides significant results: the image-based similar product retrieval model showed a "Precision at 5 " of 0.774 , with the sketch-based similar product model achieving a score of 0.445 .

Data processing research was carried out in [4-8]. Park et al. propose a software that can be applied to natural language processing (NLP) by way of pre-processing and post-processing. Pre-processing in neural machine translation (NMT) includes subword tokenization to alleviate the problem of unknown words and data augmentation to ensure that the corpus contains sufficient content. Post-processing involves automatic post editing and the application of various strategies during decoding in the translation process. The authors of this work apply various decoding strategies to Korean-English NMT, relying on a low-resource language pair. The results demonstrate that various decoding strategies perform well and present a new perspective for improving machine translation performance. Chae et al. provide a prediction methodology for particulate matter (PM) in their research [5]. Their prediction algorithm can deal with and manage symptoms such as worsened asthma, affected lungs, and various toxin-induced cancers. PM is correlated with address format, air quality data, and weather data, and the performance is measured according to the sequence length and batch size. PM prediction was also conducted with a long short-term recurrent unit (LSTM), a convolutional neural network (CNN), and a gated recurrent unit (GRU). The LSTM and GRU models yield similar prediction results, but the $\mathrm{CNN}$ suffered from the limitation of only predicting from the training data. Zhang et al. propose an optimized data sampling model to identify the status of the human brain and further discover brain activity patterns [6]. The piecewise linear approximation (SEGPA) method is applied to a segmented electroencephalography (EEG) graph. Additionally, SEGPA is integrated with normal distribution approximation (NDA) and Poisson distribution approximation (PDA). The obtained recognition system is applicable to machinery or robot control. Its performance indicates that the NDA-PDA segments with less than $10 \%$ of the original data size can achieve $98 \%$ accuracy. Woo et al. propose a smart system for extracting characteristics from computer science knowledge and organizing knowledge [7]. They enhance the efficiency and objectivity of terminology extraction by integrating a dictionary of technical terms with a hierarchical clustering structure. It is applied to high school education courses in India and Israel with several findings; the developed software can extract data at a high rate; the ratio of technical terms in the areas of architecture and organization, programming languages, and software engineering are high in advanced courses; advanced content had a high percentage of technical terms related to information systems. A three-dimensional maximum range-sum and a maximum spatiotemporal rangesum change problem are treated by Choi et al. in [8]. First, they provide a mathematical explanation for each problem and then they are resolved using several algorithms. The performance of the proposed method is verified and they also demonstrate its scalability and efficiency.

Research on analysis and management was conducted in $[9,10]$. Despite not many papers being submitted on analysis and management in this Special Issue, these two papers make important contributions. Fan et al. propose a smart enhancement scheme during decompression processing [9]. They propose two novel approaches: a two-dimensional 
F-shift transformation and a non-standard two-dimensional wavelet transformation. They test the effectiveness of the proposed scheme by demonstrating that the compression properties can be preserved and that the contrast can also be enhanced. Lim and Lee deal with the problem of computing balance in [10]. In order to solve the overloading problem in edge servers caused by sudden offloads from mobile users, a load-balancing solver is introduced for mobile devices in edge cloud computing environments. Their results show that this method improves the average CPU usage of virtual machines.

\section{Perspectives}

This Special Issue looks to the promising future of the capabilities of smart processing. The valuable findings made by the large group of contributors should be considered not only as incontestable evidence of the vibrant research activity in the field of smart processing for systems under uncertainty or perturbation but also as a symptom of the inspiring future envisioned for this knowledge area. The purpose of these papers is to share research ideas surrounding algorithm development and data processing and analysis for smart systems. We have brought 10 papers to publication after a competitive review process and encourage the related community to conduct additional research in the near future towards the adaptation of more sophisticated approaches. The papers published here will help to ensure that research continues on the distributed management of knowledge, as well as the consideration of additional types of uncertainties and perturbations.

We expect this open access forum on smart processing to represent a milestone that will encourage researchers to pursue their interest in design and manufacturing in related industrial fields. As mentioned in the Introduction, systems sustainability is required to solve the internal and external problems caused by the increased complexity and size of systems. Hence, we hope that readers will be inspired by the research ideas contained within this Special Issue and communicate with the authors regarding these important issues. As a final reflection, we would like to reflect that today it is not possible to measure the limits of this interesting research field; its horizons are near the shores of our imagination.

Author Contributions: Reviewing all papers, S.L, M.P. and E.O.; writing and correcting script; S.L. and E.O.; proofreading and checking, E.O. All authors have read and agreed to the published version of the manuscript.

Funding: Eneko Osaba would like to thank the Basque Government for its funding through the EMAITEK program.

Acknowledgments: The guest editorial team would like to thank the Editor-in-Chief and his team for their priceless help and assistance during the preparation of this Special Issue. We would also like to thank the reviewers for the insightful and valuable comments made on the submitted studies, which have been very helpful in increasing the quality of the published papers. Additionally, we would like to thank all authors and contributors.

Conflicts of Interest: The authors declare that there is no conflict of interest.

\section{References}

1. Yang, M.; Li, X.; Liu, Y. Sequence to Point Learning Based on an Attention Neural Network for Nonintrusive Load Decomposition. Electronics 2021, 10, 1657. [CrossRef]

2. Zeng, D.; He, M.-e.; Tang, X.-z.; Wang, F.-g. Cognitive Association in Interactive Evolutionary Design Process for Product Styling and Application to SUV Design. Electronics 2020, 9, 1960. [CrossRef]

3. Jo, J.; Lee, S.; Lee, C.; Lee, D.; Lim, H. Development of Fashion Product Retrieval and Recommendations Model Based on Deep Learning. Electronics 2020, 9, 508. [CrossRef]

4. Park, C.; Yang, Y.; Park, K.; Lim, H. Decoding Strategies for Improving Low-Resource Machine Translation. Electronics 2020, 9, 1562. [CrossRef]

5. Chae, M.; Han, S.; Lee, H.M. Outdoor Particulate Matter Correlation Analysis and Prediction Based Deep Learning in the Korea. Electronics 2020, 9, 1146. [CrossRef]

6. Zhang, H.L.; Lee, S.; Li, X.; He, J. EEG Self-Adjusting Data Analysis Based on Optimized Sampling for Robot Control. Electronics 2020, 9, 925. [CrossRef] 
7. Woo, H.S.; Kim, J.M.; Lee, W.G. Development of Curriculum Design Support System Based on Word Embedding and Terminology Extraction. Electronics 2020, 9, 608. [CrossRef]

8. Choi, W.; Jung, S.-Y.; Chung, J.; Hyun, K.-S.; Park, K. Scalable Algorithms for Maximizing Spatiotemporal Range Sum and Range Sum Change in Spatiotemporal Datasets. Electronics 2020, 9, 514. [CrossRef]

9. Fan, R.; Li, X.; Lee, S.; Li, T.; Zhang, H.L. Smart Image Enhancement Using CLAHE Based on an F-Shift Transformation during Decompression. Electronics 2020, 9, 1374. [CrossRef]

10. Lim, J.B.; Lee, D.W. A Load Balancing Algorithm for Mobile Devices in Edge Cloud Computing Environments. Electronics 2020, 9, 686. [CrossRef] 